\title{
A Useful Allene for the Stereoselective Synthesis of Protected
}

\section{Quaternary 2-Amino-2-Vinyl-1,3-diols}

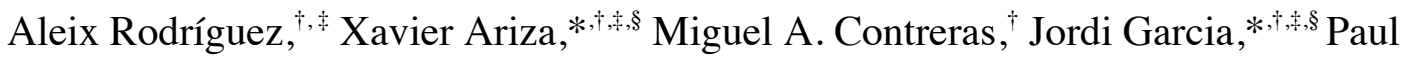 \\ Lloyd-Williams, ${ }^{\dagger,+\S}$ Nerea Mercadal,$^{\dagger}$ and Carolina Sánchez ${ }^{\dagger}$
}

†'Departament de Química Inorgànica i Orgànica, Secció de Química Orgànica, Facultat de Química, Universitat de Barcelona, Martí i Franquès 1, 08028-Barcelona, Spain

${ }^{*}$ Institut de Biomedicina de la Universitat de Barcelona (IBUB), Barcelona, Spain

${ }^{\S}$ CIBER Fisiopatología de la Obesidad y la Nutrición (CIBERobn), Instituto de Salud Carlos III, Madrid, Spain

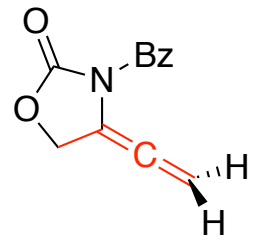

1
1) $\mathrm{Cy}_{2} \mathrm{BH}$

2) $\mathrm{RCHO}$

3) $\mathrm{N}\left(\mathrm{CH}_{2} \mathrm{CH}_{2} \mathrm{OH}\right)_{3}$

(1)<smiles>[R]C([R6])[C@]1(C=C)COC(=O)N1</smiles>

$70-90 \%$

$\mathrm{dr}>99: 1$

ABSTRACT: Treatment of readily available allene $\mathbf{1}$ with $\mathrm{Cy}_{2} \mathrm{BH}$ followed by addition of an aldehyde led to quaternary protected 2-amino-2-vinyl-1,3-diols in high yield and excellent stereochemical purity. The choice of benzoyl as $N$-protecting group is critical since the observed $N$ - to $O$-Bz transfer during the process prevents later undesired isomerizations in the adducts and keeps all heteroatoms protected.

KEYWORDS: allene, crotylborane, hydroboration, quaternary carbon, sphingosine, substituted serine

Quaternary $\alpha$ amino acids bearing adjacent hydroxyl functionalities are structural features present in numerous metabolites of biological relevance including myriocin, ${ }^{1}$ 
the proteasome inhibitor lactacystin, ${ }^{2}$ and the antitumor agent (-)-altemicidin (Fig. 1). ${ }^{3}$ Even structurally simpler $\alpha$-branched serines and threonines are also important synthetic targets. ${ }^{4}$ Furthermore, $(S)$ - $\alpha$-vinyl serine and other $\alpha$-vinyl amino acids show interesting biological activities as suicide inhibitors for enzymes of the amino acid decarboxylase class. ${ }^{5}$<smiles>N[C@@](CO)(C(=O)O)[C@@H](O)[C@H](O)C/C=C/CCCCCCC(=O)c1ccccc1</smiles>

(+)-Myriocin<smiles>CNC(=O)CCSC(=O)[C@@]1(C(O)C(C)C)NC(=O)[C@H](C)[C@H]1O</smiles>

$(-)$-Altemicidin

(+)-Lactacystin<smiles>C=C[C@](N)(CO)C(=O)O</smiles><smiles>[R]C(N)C([R])O</smiles>

(S)- $\alpha$-Vinyl Serine

$\alpha$-Branched Threonine

Figure 1. Compounds incorporating quaternary $\beta$-hydroxylated $\alpha$-amino acids

Sphingolipids are a family of lipids that play essential roles as structural cell membrane components and also in cell signalling through a complex metabolism catalyzed by specific enzymes. ${ }^{6}$ From a structural point of view, most mammalian sphingolipids share a common backbone incorporating (E)-2-amino-4-octadecen-1,3-diol (sphingosine). Interestingly, structural analogues of sphingosine such as quaternary 2vinyl compound $\mathbf{2}$ might act as selective inhibitors of these enzymes. ${ }^{6 c}$ 


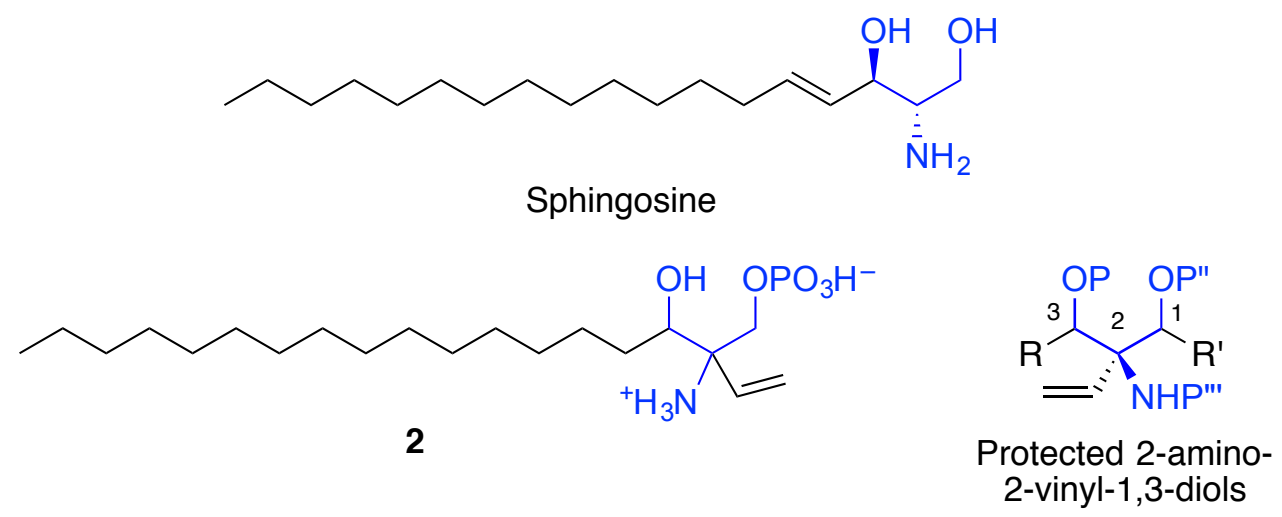

Figure 2. Sphingosine, compound 2 and its possible precursor

We envisaged that both $\beta$-hydroxylated $\alpha$-amino acids such as $(S)$ - $\alpha$-vinyl serine and certain analogues of sphingolipids such as compound $\mathbf{2}$, might be generated from protected 2-amino-2-vinyl-1,3-diols (Figure 2). ${ }^{7}$

As part of our studies aimed at the synthesis of polyhydroxylated $\alpha$-amino acids, ${ }^{8}$ we recently reported a new tandem reaction leading to protected tosylcarbamates $\mathbf{3}$ in high yields and excellent diastereoselectivities (Scheme 1). ${ }^{9}$ This simple one-pot process was based on the hydroboration of tosyl allene 4 with $\mathrm{Cy}_{2} \mathrm{BH}$, followed by the addition of an aldehyde. 


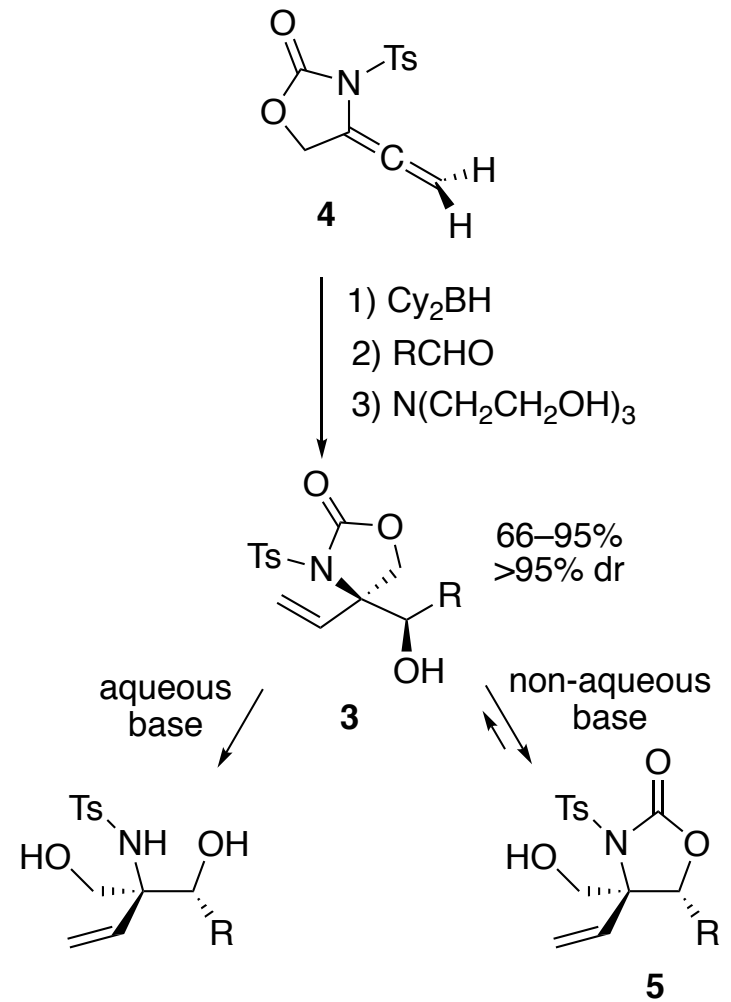

Scheme 1. Preparation of $N$-tosylcarbamates 3 and their hydrolysis and isomerization to 5

However, in practice this procedure was hampered by drawbacks related to the use of the $N$-tosyl protecting group. This enhances the electrophilic character of the carbonyl group in 4 favoring hydrolysis of the carbamate or its isomerization to $\mathrm{N}$ tosylcarbamates $\mathbf{5}$ in aqueous or dry basic media, respectively. Unfortunately, these deleterious side reactions have also been observed ${ }^{10}$ occasionally during work-up and/or chromatographic purification of compounds 3 . On the other hand, removing the robust tosyl group in the final steps of a multi-step synthetic sequence could be troublesome limiting the synthetic utility of the methodology.

Some of these drawbacks were encountered in our approach to the synthesis of vinylsphinganines such as $\mathbf{2}$, potential inhibitors of sphingosine-1-phosphate lyase. We envisaged that allene 1, easily obtained from 2-butyn-1,4-diol in 61\% yield (Scheme 
$2)^{11}$ with the easily removable benzoyl $\mathrm{N}$-protecting group, might be a better choice since it should be bulky enough to promote high stereoselectivity in the hydroboration step.

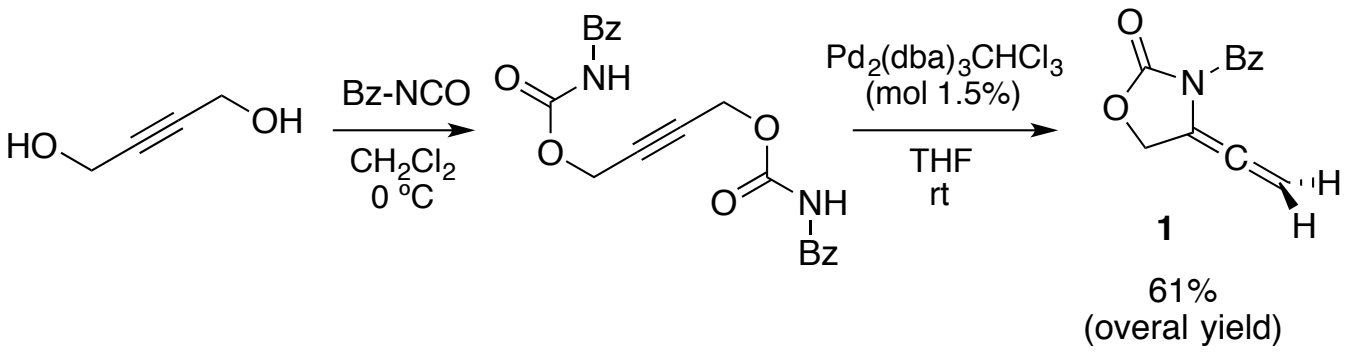

Scheme 2. Synthesis of allene 1 from 2-butyn-1,4-diol

When allene 1 was hydroborated with dicyclohexylborane in $\mathrm{CH}_{2} \mathrm{Cl}_{2}$ followed by addition of isobutyraldehyde and treatment with triethanolamine, carbamate $6 \mathbf{a}$ was isolated in $78 \%$ yield (Scheme 3). As expected, a single diastereoisomer was obtained. Presumably, the relative stereochemistry of $\mathbf{6 a}$ arises from the hydroboration of the less hindered face of allene 1 to afford the corresponding (Z)-2-alkenylborane that is added to the aldehyde through a six-membered transition state where 1,3-axial interactions are minimized. Surprisingly, $\mathrm{N}$ to $\mathrm{O}$ migration of the benzoyl group in carbamate $\mathbf{6 a}$ had also occurred. ${ }^{12}$ A corresponding migration was not observed when tosylated allene 4 was subjected to similar transformations. As expected, protected carbamate 6a was stable under basic, non-nucleophilic conditions (DBU) and the ring isomerization observed in the case of tosylcarbamates $\mathbf{5}$ did not occur with the benzoyl derivative. 
<smiles>C=C=C1COC(=O)N1C(=O)c1ccccc1</smiles>
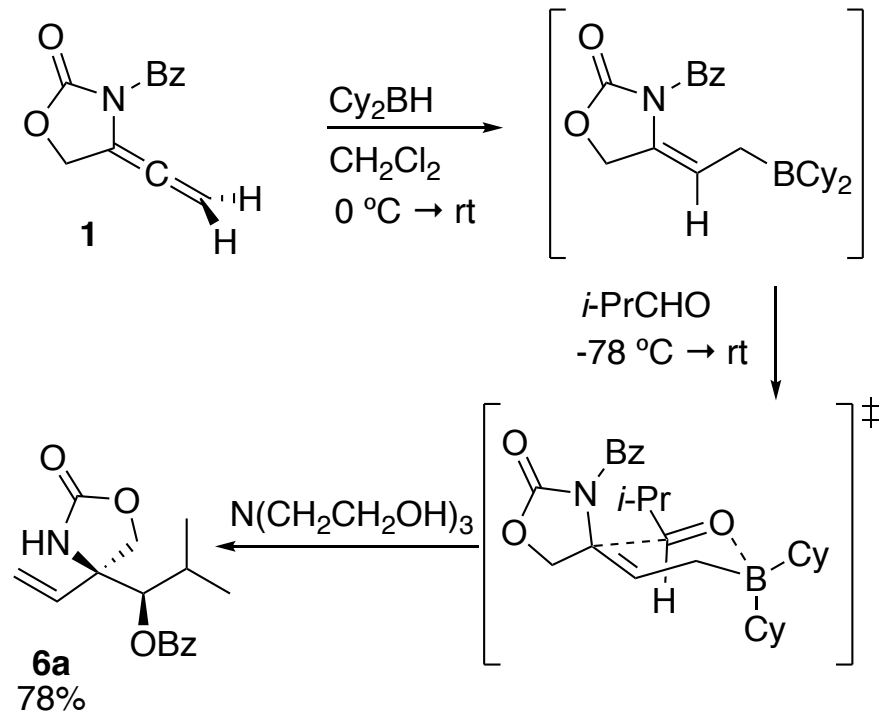

Scheme 3. Addition of allene 1 to isobutyraldehyde

The reaction was repeated with a variety of aldehydes (Table 1), in order to explore its scope. Carbamates 6 were obtained in good yields for aliphatic (entries 1 and 2), aromatic (entry 3), $\alpha, \beta$-alkenyl (entries 4 and 5) and $\alpha, \beta$-alkynyl aldehydes (entry 6). In all cases $\mathrm{N}$ to $\mathrm{O}$ migration of the benzoyl group was observed. Furthermore, a single diastereomer ${ }^{13}$ was always obtained including in the cases of those carbamates that might be useful in the synthesis of SPL inhibitors (entries 2, 5 and 6).
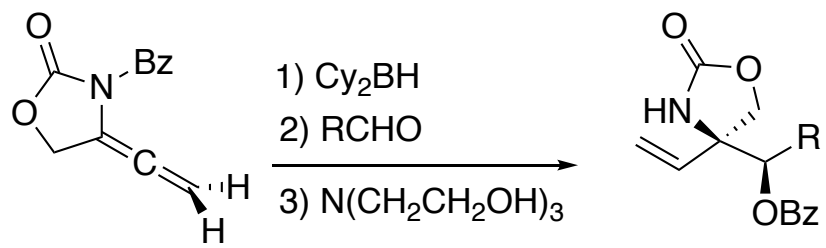

\begin{tabular}{|c|c|c|c|}
\hline Entry & $\mathrm{R}$ & Product & Yield \\
\hline 1 & ethyl & $\mathbf{6 b}$ & $81 \%$ \\
\hline 2 & dodecanyl & $\mathbf{6 c}$ & $69 \%$ \\
\hline 3 & phenyl & $\mathbf{6 d}$ & $82 \%$ \\
\hline 4 & vinyl & $\mathbf{6 e}$ & $84 \%$ \\
\hline
\end{tabular}




\begin{tabular}{|c|c|c|c|}
\hline 5 & $(E)$-1-undecenyl & $\mathbf{6 f}$ & $74 \%$ \\
\hline 6 & 1-heptynyl & $\mathbf{6 g}$ & $90 \%$ \\
\hline
\end{tabular}

Table 1. Addition of allene 1 to aldehydes

Enantiopure carbamates were obtained when the reaction was performed with chiral aldehydes that exhibited strong stereofacial selectivity such as those derived from $(R)$ glyceraldehyde or (R)-lactaldehyde (Scheme 4). Again, only one stereoisomer of $\mathbf{6 h}$ and 6i were obtained in good yield with complete migration of the benzoyl group.
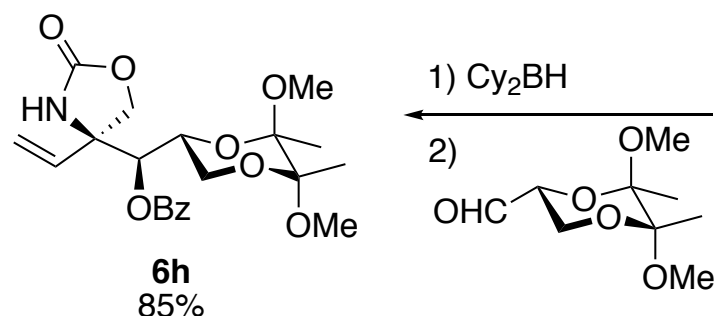

3) $\mathrm{N}\left(\mathrm{CH}_{2} \mathrm{CH}_{2} \mathrm{OH}\right)_{3}$

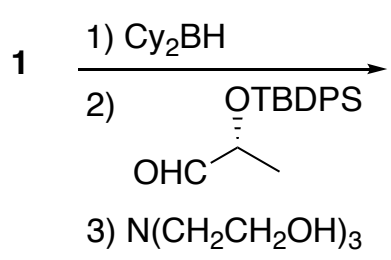

3) $\mathrm{N}\left(\mathrm{CH}_{2} \mathrm{CH}_{2} \mathrm{OH}\right)_{3}$

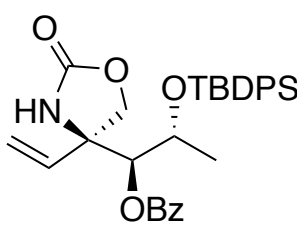

$8 \mathbf{6}$

Scheme 4. Addition of allene 1 to enantiopure aldehydes.

Compound 6i was used to check the deprotection steps in the synthesis of sphingosine derivatives such as myriocin (Figure 1) and we confirmed that this fully orthogonally protected carbamate can be partially deprotected (Scheme 5). Thus, treatment of carbamate $6 \mathbf{i}$ with $\mathrm{K}_{2} \mathrm{CO}_{3}$ in $\mathrm{MeOH}$ removed the benzoyl group (86\% yield) with only partial migration $(10 \%)$ of the TBDPS group to the oxygen atom previously protected by the benzoyl group. Complete removal of the TBDPS group was achieved by treatment with TBAF and afforded carbamate 7 (78\% yield). In the absence of the tosyl group, concomitant isomerization of the carbamate, as occurred when it was present (see Scheme 1), was minimized. 


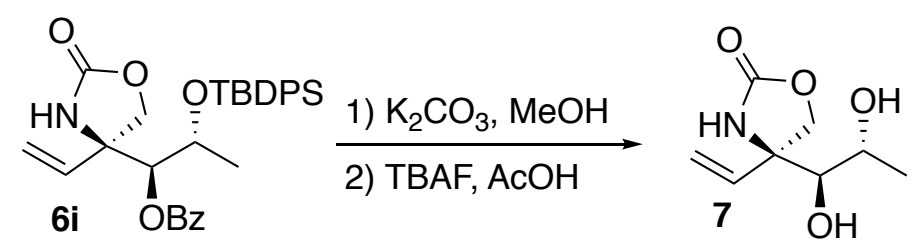

Scheme 5. Deprotection of $6 \mathbf{i}$ to give 7

In summary, allene $\mathbf{1}$ is a useful starting material for the synthesis of protected quaternary 2-amino-2-vinyl-1,3-diols by hydroboration followed by aldehyde addition since it avoids undesired migrations and affords a fully protected carbamate with complete stereoselectivity from a range of aldehydes in one step.

\section{Experimental Section}

All reactions involving moisture- or air-sensitive reagents were performed in oven-dried glassware under $\mathrm{N}_{2}$. Chemical shifts $(\delta)$ are quoted in parts per million and in ${ }^{1} \mathrm{H}$ NMR are referenced to internal TMS (for $\left.\mathrm{CDCl}_{3}\right) \cdot{ }^{13} \mathrm{C} \mathrm{NMR}$ are referenced to $\mathrm{CDCl}_{3}(\delta 77.0$ ppm). Column chromatography was performed on silica gel (Merck 230-400 mesh). HRMS analyses were recorded on a LC/MSD-TOF mass spectrometer. Chiral aldehydes were prepared from D-mannitol ${ }^{14}$ and $(R)$-methyl lactate. ${ }^{15}$

3-Benzoyl-4-vinylideneoxazolidin-2-one (1). A solution of benzoyl isocyanate $(4.20 \mathrm{~g}$, $25.6 \mathrm{mmol})$ in anhydrous $\mathrm{CH}_{2} \mathrm{Cl}_{2}(20 \mathrm{~mL})$ was added to 2-butyn-1,4-diol (1.00 g, 11.6 mmol) at $0{ }^{\circ} \mathrm{C}$ under $\mathrm{N}_{2}$ atmosphere. The mixture was stirred for 5 hours at $\mathrm{rt}$ and the solvent was removed. A solution of $\mathrm{Pd}_{2}(\mathrm{dba})_{3} \cdot \mathrm{CHCl}_{3}(0.055 \mathrm{~g}, 0.05 \mathrm{mmol})$ in anhydrous THF $(40 \mathrm{~mL})$ and triethylamine $(0.087 \mathrm{~mL}, 0.64 \mathrm{mmol})$ were added under $\mathrm{N}_{2}$ atmosphere. The mixture was stirred for $16 \mathrm{~h}$ at $\mathrm{rt}$ and filtered through a pad of Celite. The solid was washed with EtOAc. The solvent was removed and the crude residue was 
purified by column chromatography (hexanes/EtOAc 4:1) to afford $0.595 \mathrm{~g}(61 \%)$ of allene 1: yellow solid; mp 102-103 ${ }^{\circ} \mathrm{C}$ (lit. $\left.{ }^{11} 101.2-103{ }^{\circ} \mathrm{C}\right) ; R_{f}$ (hexanes/EtOAc 2:1) = 0.35; ${ }^{1} \mathrm{H}$ NMR (400 MHz, $\left.\mathrm{CDCl}_{3}\right) \delta 7.68(\mathrm{~m}, 2 \mathrm{H}), 7.57(\mathrm{~m}, 1 \mathrm{H}), 7.45(\mathrm{~m}, 2 \mathrm{H}), 5.57(\mathrm{t}, J$ $=4.7 \mathrm{~Hz}, 2 \mathrm{H}), 5.04(\mathrm{t}, J=4.7 \mathrm{~Hz}, 2 \mathrm{H}) ;{ }^{13} \mathrm{C} \mathrm{NMR}\left(101 \mathrm{MHz}, \mathrm{CDCl}_{3}\right) \delta 193.6,167.3$, 151.9, 132.8, 129.2, 128.1, 128.0, 103.9, 90.4, 63.9; IR (film): 1792, 1689, 1331, 1308, 1157, $1068 \mathrm{~cm}^{-1}$; HRMS (ESI+) calculated for $\mathrm{C}_{12} \mathrm{H}_{10} \mathrm{NO}_{3}[\mathrm{M}+\mathrm{H}]^{+}=216.0655$, found $=$ 216.0651.

\section{General procedure for the allene hydroboration-aldehyde addition tandem process}

A solution of the allene (1.00 equiv, $1.20 \mathrm{mM}$ ) in anhydrous $\mathrm{CH}_{2} \mathrm{Cl}_{2}$ was added to a suspension of $\mathrm{Cy}_{2} \mathrm{BH}$ (1.20 equiv, $1.40 \mathrm{mM}$ ) in $\mathrm{CH}_{2} \mathrm{Cl}_{2}$ at $0{ }^{\circ} \mathrm{C}$ and under nitrogen atmosphere. The resulting mixture was stirred for $10 \mathrm{~min}$ at $0{ }^{\circ} \mathrm{C}$ and for $1 \mathrm{~h}$ at $\mathrm{rt}$. The resulting solution was then cooled to $-78^{\circ} \mathrm{C}$, and the aldehyde (1.40 equiv) was added. The reaction was stirred for $4 \mathrm{~h}$ at $\mathrm{rt}$, and was then quenched by addition of triethanolamine (2.50 equiv). The resulting mixture was stirred for $1 \mathrm{~h}$ at $\mathrm{rt}$. Solvent removal gave a crude that was purified by flash column cromatography yielding the following adducts:

(RS)-2-Methyl-1-[(SR)-2-oxo-4-vinyloxazolidin-4-yl]propyl benzoate (6a), $0.221 \mathrm{~g}$ $(78 \%)$ from $0.210 \mathrm{~g}(0.98 \mathrm{mmol})$ of allene $\mathbf{1}$ as a colorless oil; $R_{f}($ hexanes $/$ EtOAc $7: 3)=$ 0.20; ${ }^{1} \mathrm{H} \mathrm{NMR}\left(400 \mathrm{MHz}, \mathrm{CDCl}_{3}\right) \delta 8.04(\mathrm{~d}, J=7.6 \mathrm{~Hz}, 2 \mathrm{H}), 7.61(\mathrm{t}, J=7.6 \mathrm{~Hz}, 1 \mathrm{H})$, $7.48(\mathrm{t}, J=7.6 \mathrm{~Hz}, 2 \mathrm{H}), 6.09(\mathrm{dd}, J=17.3,10.6 \mathrm{~Hz}, 1 \mathrm{H}), 5.96(\mathrm{bs}, 1 \mathrm{H}), 5.48(\mathrm{~d}, J=$ $17.3 \mathrm{~Hz}, 1 \mathrm{H}), 5.40(\mathrm{~d}, J=10.6 \mathrm{~Hz}, 1 \mathrm{H}), 5.20(\mathrm{~d}, J=4.2 \mathrm{~Hz}, 1 \mathrm{H}), 4.53(\mathrm{~d}, J=8.6 \mathrm{~Hz}$, $1 \mathrm{H}), 4.18(\mathrm{~d}, J=8.6 \mathrm{~Hz}, 1 \mathrm{H}), 2.13(\mathrm{~m}, 1 \mathrm{H}), 1.00(\mathrm{~d}, J=7.4 \mathrm{~Hz}, 3 \mathrm{H}), 1.00(\mathrm{~d}, J=7.4$ $\mathrm{Hz}, 3 \mathrm{H}) ;{ }^{13} \mathrm{C}$ NMR $\left(101 \mathrm{MHz}, \mathrm{CDCl}_{3}\right) \delta 166.1,158.7,136.2,133.5,129.7,129.2,128.6$, 116.6, 79.5, 72.3, 65.0, 29.3, 20.8, 17.5; IR (film): 3334, 2968, 2933, 1759, $1735 \mathrm{~cm}^{-1}$; HRMS (ESI+) calculated for $\mathrm{C}_{16} \mathrm{H}_{20} \mathrm{NO}_{4}[\mathrm{M}+\mathrm{H}]^{+}=290.1387$, found $=290.1386$. 


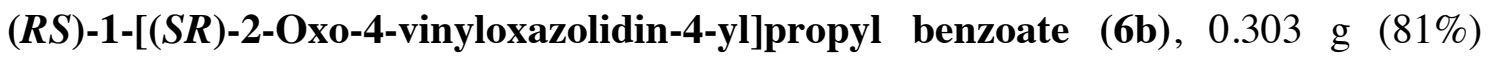
from $0.293 \mathrm{~g}(1.37 \mathrm{mmol})$ of allene 1 as a colorless oil; $R_{f}($ hexanes $/$ EtOAc $7: 3)=0.20$; ${ }^{1} \mathrm{H} \mathrm{NMR}\left(400 \mathrm{MHz}, \mathrm{CDCl}_{3}\right) \delta 8.06(\mathrm{t}, J=7.7 \mathrm{~Hz}, 2 \mathrm{H}), 7.58(\mathrm{t}, J=7.7 \mathrm{~Hz}, 1 \mathrm{H}), 7.47$ (bs, 1H), $7.45(\mathrm{t}, J=7.7 \mathrm{~Hz}, 2 \mathrm{H}), 6.03(\mathrm{dd}, J=17.3,10.7 \mathrm{~Hz}, 1 \mathrm{H}), 5.46(\mathrm{~d}, J=17.3 \mathrm{~Hz}$, $1 \mathrm{H}), 5.33(\mathrm{~d}, J=10.7 \mathrm{~Hz}, 1 \mathrm{H}), 5.28(\mathrm{dd}, J=8.0,2.0 \mathrm{~Hz}, 1 \mathrm{H}), 4.46(\mathrm{~d}, J=8.8 \mathrm{~Hz}, 1 \mathrm{H})$, $4.16(\mathrm{~d}, J=8.8 \mathrm{~Hz}, 1 \mathrm{H}), 1.81-1.64(\mathrm{~m}, 2 \mathrm{H}), 0.95(\mathrm{t}, J=7.4 \mathrm{~Hz}, 3 \mathrm{H}) ;{ }^{13} \mathrm{C} \mathrm{NMR}(101$ $\left.\mathrm{MHz}, \mathrm{CDCl}_{3}\right) \delta 166.1,159.6,135.6,133.3,129.7,129.2,128.5,116.9,77.8,72.4,64.8$, 22.5, 10.1; IR (film): 3253, 2924, 2854, 1756, 1719, 1268, $1106 \mathrm{~cm}^{-1}$; HRMS (ESI+) calculated for $\mathrm{C}_{15} \mathrm{H}_{18} \mathrm{NO}_{4}[\mathrm{M}+\mathrm{H}]^{+}=276.1230$, found $=276.1232$.

$(\boldsymbol{R S})-1-[(\boldsymbol{S R})-2-0 x 0-4-v i n y l o x a z o l i d i n-4-y l]$ tridecyl benzoate (6c), $0.461 \mathrm{~g}(69 \%)$ from $0.348 \mathrm{~g}(1.62 \mathrm{mmol})$ of allene 1 as a colorless oil; $R_{f}($ hexanes/EtOAc $3: 2)=0.74$; ${ }^{1} \mathrm{H}$ NMR $\left(400 \mathrm{MHz}, \mathrm{CDCl}_{3}\right) \delta$ 8.03-7.97 (m, 2H), 7.62-7.54 (m, 1H), 7.49-7.41 (m, 2H), $6.00(\mathrm{dd}, J=17.2,10.7 \mathrm{~Hz}, 1 \mathrm{H}), 5.83(\mathrm{bs}, 1 \mathrm{H}), 5.42(\mathrm{~d}, J=17.2 \mathrm{~Hz}, 1 \mathrm{H}), 5.35(\mathrm{~d}, J=$ $10.7 \mathrm{~Hz}, 1 \mathrm{H}), 5.28(\mathrm{dd}, J=8.9,4.3 \mathrm{~Hz}, 1 \mathrm{H}), 4.45(\mathrm{~d}, J=8.7 \mathrm{~Hz}, 1 \mathrm{H}), 4.15(\mathrm{~d}, J=8.7$ $\mathrm{Hz}, 1 \mathrm{H}), 1.70-1.58(\mathrm{~m}, 2 \mathrm{H}), 1.37-1.16(\mathrm{~m}, 20 \mathrm{H}), 0.86(\mathrm{t}, J=6.9 \mathrm{~Hz}, 3 \mathrm{H}) ;{ }^{13} \mathrm{C} \mathrm{NMR}$ $\left(101 \mathrm{MHz}, \mathrm{CDCl}_{3}\right) \delta 166.3,158.7,136.2,133.6,129.9,129.4,128.7,117.2,76.2,72.0$, $64.8,35.7,32.1,29.8,29.7,29.6,29.5,29.5,29.4,25.8,25.6,22.8,14.3$; IR (ATR): 3205, 2922, 2852, 1755, 1718, 1264, 1095, $709 \mathrm{~cm}^{-1}$; HRMS (ESI+) calculated for $\mathrm{C}_{25} \mathrm{H}_{38} \mathrm{NO}_{4}[\mathrm{M}+\mathrm{H}]^{+}=416.2795$, found $=416.2789$.

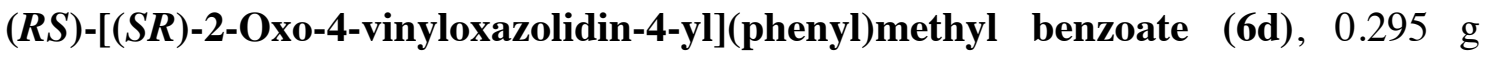
$(82 \%)$ from $0.240 \mathrm{~g}(1.11 \mathrm{mmol})$ of allene 1 as a colorless solid; $\mathrm{mp}=72-74{ }^{\circ} \mathrm{C} ; R_{f}$ $\left(\right.$ hexanes/EtOAc 7:3) $=0.18 ;{ }^{1} \mathrm{H}$ NMR $\left(400 \mathrm{MHz}, \mathrm{CDCl}_{3}\right) \delta 8.08(\mathrm{~d}, J=8.0 \mathrm{~Hz}, 2 \mathrm{H})$, 7.56-7.34 (m, 9H), $6.07(\mathrm{dd}, J=18.0,9.9 \mathrm{~Hz}, 1 \mathrm{H}), 6.06(\mathrm{~s}, 1 \mathrm{H}), 5.38(\mathrm{~d}, J=18.0 \mathrm{~Hz}$, $1 \mathrm{H}), 5.35(\mathrm{~d}, J=9.9 \mathrm{~Hz}, 1 \mathrm{H}), 4.57(\mathrm{~d}, J=8.8 \mathrm{~Hz}, 1 \mathrm{H}), 4.17(\mathrm{~d}, J=8.8 \mathrm{~Hz}, 1 \mathrm{H}) ;{ }^{13} \mathrm{C}$ $\operatorname{NMR}\left(101 \mathrm{MHz}, \mathrm{CDCl}_{3}\right) \delta 165.1,159.3,135.9,134.5,133.4,129.7,129.1,129.0$, 
128.4, 127.7, 117.1, 78.2, 71.8, 65.0; IR (KBr): 3462, 3215, 1753, 1712, $1269 \mathrm{~cm}^{-1}$; HRMS (ESI+) calculated for $\mathrm{C}_{19} \mathrm{H}_{18} \mathrm{NO}_{4}[\mathrm{M}+\mathrm{H}]^{+}=324.1230$, found $=324.1230$.

$(\boldsymbol{R S})$-1-[(SR)-2-Oxo-4-vinyloxazolidin-4-yl]allyl benzoate (6e), $0.282 \mathrm{~g}(84 \%)$ from $0.263 \mathrm{~g}(1.22 \mathrm{mmol})$ of allene 1 as a yellow oil; $R_{f}$ (hexanes/EtOAc 7:3) $=0.21 ;{ }^{1} \mathrm{H}$ NMR (400 MHz, $\left.\mathrm{CDCl}_{3}\right) \delta 8.04(\mathrm{t}, J=7.8 \mathrm{~Hz}, 2 \mathrm{H}), 7.59(\mathrm{t}, J=7.8 \mathrm{~Hz}, 1 \mathrm{H}), 7.49(\mathrm{t}, J=$ $7.8 \mathrm{~Hz}, 2 \mathrm{H}), 6.41(\mathrm{bs}, 1 \mathrm{H}), 6.03(\mathrm{dd}, J=17.3,10.7 \mathrm{~Hz}, 1 \mathrm{H}), 5.87$ (ddd $, J=17.3,10.7$, $7.0 \mathrm{~Hz}, 1 \mathrm{H}), 5.57(\mathrm{~d}, J=7.0 \mathrm{~Hz}, 1 \mathrm{H}), 5.56(\mathrm{~d}, J=0.8 \mathrm{~Hz}, 1 \mathrm{H}), 5.54(\mathrm{~d}, J=17.3 \mathrm{~Hz}$, $1 \mathrm{H}), 5.46(\mathrm{~d}, J=17.3 \mathrm{~Hz}, 1 \mathrm{H}), 5.46(\mathrm{~d}, J=10.7 \mathrm{~Hz}, 1 \mathrm{H}), 4.50(\mathrm{~d}, J=8.8 \mathrm{~Hz}, 1 \mathrm{H}), 4.17$ $(\mathrm{d}, J=8.8 \mathrm{~Hz}, 1 \mathrm{H}) ;{ }^{13} \mathrm{C} \mathrm{NMR}\left(101 \mathrm{MHz}, \mathrm{CDCl}_{3}\right) \delta 165.1,159.4,135.5,133.4,130.1$, 129.7, 129.2, 128.5, 122.0, 117.4, 77.4, 71.8, 64.1; IR (film): 3250, 1756, 1721, 1266, $1070 \mathrm{~cm}^{-1}$; HRMS (ESI+) calculated for $\mathrm{C}_{14} \mathrm{H}_{16} \mathrm{NO}_{2}[\mathrm{M}+\mathrm{H}]^{+}=230.1176$, found $=$ 230.1176.

$(R S, E)-1-[(S R)-2-O x 0-4-v i n y l o x a z o l i d i n-4-y l] d o d e c-2-e n-1-y l$ benzoate $(6 f), 0.331 \mathrm{~g}$ $(74 \%)$ from $0.243 \mathrm{~g}(1.13 \mathrm{mmol})$ of allene 1 as a colorless oil; $R_{f}$ (hexanes/EtOAc 4:1)= 0.22; ${ }^{1} \mathrm{H}$ NMR $\left(400 \mathrm{MHz}, \mathrm{CDCl}_{3}\right) \delta$ 8.04-7.98 (m, 2H), 7.59-7.53 (m, 1H), 7.46-7.41 $(\mathrm{m}, 2 \mathrm{H}), 6.30(\mathrm{bs}, 1 \mathrm{H}), 6.05-5.96(\mathrm{~m}, 2 \mathrm{H}), 5.54-5.41(\mathrm{~m}, 3 \mathrm{H}), 5.35(\mathrm{~d}, J=10.7 \mathrm{~Hz}, 1 \mathrm{H})$, $4.46(\mathrm{~d}, J=8.7 \mathrm{~Hz}, 1 \mathrm{H}), 4.14(\mathrm{~d}, J=8.7 \mathrm{~Hz}, 1 \mathrm{H}), 2.05(\mathrm{dt}, J=7.8,4.0 \mathrm{~Hz},=2 \mathrm{H}), 1.40-$ $1.31(\mathrm{~m}, 2 \mathrm{H}), 1.30-1.19(\mathrm{~m}, 12 \mathrm{H}), 0.86(\mathrm{t}, J=6.9 \mathrm{~Hz}, 3 \mathrm{H}) ;{ }^{13} \mathrm{C} \mathrm{NMR}\left(101 \mathrm{MHz}, \mathrm{CDCl}_{3}\right)$ $\delta 165.4,159.1,140.7,136.2,133.5,129.8,129.7,128.7,121.8,117.3,77.8,72.2,64.3$, 32.6, 32.0, 29.6, 29.5, 29.4, 29.3, 28.8, 22.8, 14.2; IR (ATR): 3240, 2923, 1755, 1707, 1263, $709 \mathrm{~cm}^{-1}$; HRMS (ESI+) calculated for $\mathrm{C}_{24} \mathrm{H}_{37} \mathrm{~N}_{2} \mathrm{O}_{4}\left[\mathrm{M}+\mathrm{NH}_{4}\right]^{+}=417.2748$, found $=417.2747$.

(RS)-1-[(SR)-2-Oxo-4-vinyloxazolidin-4-yl]oct-2-yn-1-yl benzoate $\quad(6 \mathrm{~g}) .0 .340 \mathrm{~g}$ $(90 \%)$ from $0.237 \mathrm{~g}(1.10 \mathrm{mmol})$ of allene 1 as a colorless solid; $\mathrm{mp}=122-124{ }^{\circ} \mathrm{C} ; R_{f}$ (hexanes/EtOAc 4:1) = 0.22; ${ }^{1} \mathrm{H}$ NMR $\left(400 \mathrm{MHz} \mathrm{CDCl}_{3}\right) \delta 8.02(\mathrm{~m}, 2 \mathrm{H}), 7.59(\mathrm{~m}, 1 \mathrm{H})$, 
$7.45(\mathrm{~m}, 2 \mathrm{H}), 6.10(\mathrm{dd}, J=17.2,10.7 \mathrm{~Hz}, 1 \mathrm{H}), 5.79(\mathrm{bs}, 1 \mathrm{H}), 5.65(\mathrm{t}, J=2.0 \mathrm{~Hz}, 1 \mathrm{H})$, $5.50(\mathrm{~d}, J=17.2 \mathrm{~Hz}, 1 \mathrm{H}), 5.39(\mathrm{~d}, J=10.7 \mathrm{~Hz}, 1 \mathrm{H}), 4.70(\mathrm{~d}, J=8.8 \mathrm{~Hz}, 1 \mathrm{H}), 4.20(\mathrm{~d}, J$ $=8.8 \mathrm{~Hz}, 1 \mathrm{H}), 2.21(\mathrm{dt}, J=7.1,2.0 \mathrm{~Hz}, 2 \mathrm{H}), 1.53-1.48(\mathrm{~m}, 2 \mathrm{H}), 1.35-1.27(\mathrm{~m}, 4 \mathrm{H}), 0.88$ $(\mathrm{t}, J=7.0 \mathrm{~Hz}, 3 \mathrm{H}) ;{ }^{13} \mathrm{C}$ NMR $\left(101 \mathrm{MHz}, \mathrm{CDCl}_{3}\right) \delta 165.2,147.3,135.2,133.6,130.9$, 129.0, 128.5, 117.6, 89.7, 73.1, 72.0, 67.8, 64.4, 31.0, 27.8, 22.0, 18.6, 13.9; IR (KBr): $3243,2932,2860,1760,1684 \mathrm{~cm}^{-1}$; HRMS (ESI+) calculated for $\mathrm{C}_{20} \mathrm{H}_{24} \mathrm{NO}_{4}[\mathrm{M}+\mathrm{H}]^{+}=$ 342.1700 , found $=342.1696$.

$(S)-[(2 R, 5 R, 6 R)-5,6-D i m e t h o x y-5,6-d i m e t h y l-1,4-d i o x a n-2-y l][(S)-2-0 x o-4-$

vinyloxazolidin-4-yl]methyl benzoate $(6 \mathbf{h}), 0.448 \mathrm{~g}(85 \%)$ from $0.269 \mathrm{~g}(1.25 \mathrm{mmol})$ of allene 1 as a colorless solid; $\mathrm{mp}=69-71{ }^{\circ} \mathrm{C} ; R_{f}($ hexanes $/$ EtOAc $7: 3)=0.16 ;[\alpha]_{\mathrm{D}}{ }^{25}-$ $81.9\left(c=1.0, \mathrm{CHCl}_{3}\right) ;{ }^{1} \mathrm{H} \mathrm{NMR}\left(400 \mathrm{MHz}, \mathrm{CDCl}_{3}\right) \delta 7.98(\mathrm{~d}, J=7.9 \mathrm{~Hz}, 2 \mathrm{H}), 7.63(\mathrm{~d}, J$ $=7.9 \mathrm{~Hz}, 1 \mathrm{H}), 7.48(\mathrm{~d}, J=7.9 \mathrm{~Hz}, 2 \mathrm{H}), 6.27(\mathrm{dd}, J=17.2,10.6 \mathrm{~Hz}, 1 \mathrm{H}), 5.85(\mathrm{bs}, 1 \mathrm{H})$, $5.59(\mathrm{~d}, J=17.2 \mathrm{~Hz}, 1 \mathrm{H}), 5.48(\mathrm{~d}, J=10.6 \mathrm{~Hz}, 1 \mathrm{H}), 5.33(\mathrm{~d}, J=8.7 \mathrm{~Hz}, 1 \mathrm{H}), 4.36(\mathrm{~d}, J$ $=8.7 \mathrm{~Hz}, 1 \mathrm{H}), 4.31-4.23(\mathrm{~m}, 1 \mathrm{H}), 4.05(\mathrm{~d}, J=8.7 \mathrm{~Hz}, 1 \mathrm{H}), 3.73(\mathrm{t}, J=11.4 \mathrm{~Hz}, 1 \mathrm{H})$, $3.40(\mathrm{dd}, J=11.4,3.4 \mathrm{~Hz}, 1 \mathrm{H}), 3.27(\mathrm{~s}, 3 \mathrm{H}), 3.25(\mathrm{~s}, 3 \mathrm{H}), 1.32(\mathrm{~s}, 3 \mathrm{H}), 1.25(\mathrm{~s}, 3 \mathrm{H}) ;{ }^{13} \mathrm{C}$ NMR (101 MHz, $\left.\mathrm{CDCl}_{3}\right) \delta 165.1,158.1,135.6,134.1,129.8,128.3,127.6,117.2,99.6$, 98.1, 74.7, 73.7, 67.5, 63.3, 60.8, 49.2, 48.2, 17.5, 17.5; IR (KBr): 3342, 2991, 2949, 1763, $1726 \mathrm{~cm}^{-1}$; HRMS (ESI+) calculated for $\mathrm{C}_{21} \mathrm{H}_{28} \mathrm{NO}_{8}[\mathrm{M}+\mathrm{H}]^{+}=422.1809$, found $=$ 422.1806.

$(1 S, 2 R)-2-[($ tert-Butyldiphenylsilyl)oxy]-1-[(S)-2-oxo-4-vinyloxazolidin-4-yl]propyl benzoate (6i), $0.673 \mathrm{~g}(84 \%)$ from $0.324 \mathrm{~g}(1.51 \mathrm{mmol})$ of allene 1 as a colorless oil; $R_{f}$ $\left(\right.$ hexanes/EtOAc 4:1) $=0.24 ;[\alpha]_{\mathrm{D}}{ }^{25}+31.7\left(c=1.0, \mathrm{CHCl}_{3}\right) ;{ }^{1} \mathrm{H} \mathrm{NMR}\left(400 \mathrm{MHz}, \mathrm{CDCl}_{3}\right)$ $\delta 8.00(\mathrm{~m}, 2 \mathrm{H}), 7.60-7.71(\mathrm{~m}, 5 \mathrm{H}), 7.35-7.50(\mathrm{~m}, 8 \mathrm{H}), 6.05(\mathrm{dd}, J=17.3,10.7 \mathrm{~Hz}, 1 \mathrm{H})$, $5.59(\mathrm{bs}, 1 \mathrm{H}), 5.44(\mathrm{~d}, J=17.3 \mathrm{~Hz}, 1 \mathrm{H}), 5.39(\mathrm{~d}, J=10.7 \mathrm{~Hz}, 1 \mathrm{H}), 5.30(\mathrm{~m}, 1 \mathrm{H}), 4.41$ $(\mathrm{d}, J=8.7 \mathrm{~Hz}, 1 \mathrm{H}), 4.15(\mathrm{~m}, 1 \mathrm{H}), 4.07(\mathrm{~d}, J=8.7 \mathrm{~Hz}, 1 \mathrm{H}), 1.06(\mathrm{~s}, 9 \mathrm{H}), 1.03(\mathrm{~d}, J=6.3$ 
$\mathrm{Hz}, 3 \mathrm{H}) ;{ }^{13} \mathrm{C} \mathrm{NMR}\left(101 \mathrm{MHz}, \mathrm{CDCl}_{3}\right) \delta 158.8,135.9,135.7,135.6,133.5,132.7,130.1$, 129.9, 127.9, 127.7, 115.8, 78.7, 73.6, 70.7, 63.6, 27.0, 19.1, 18.9; IR (film): 3250, $3070,2931,2858,1759,1728 \mathrm{~cm}^{-1}$; HRMS (ESI+) calculated for $\mathrm{C}_{31} \mathrm{H}_{36} \mathrm{NO}_{5} \mathrm{Si}[\mathrm{M}+\mathrm{H}]^{+}$ $=530.2356$, found $=530.2357$.

(S)-4-[(1S,2R)-1,2-Dihydroxypropyl]-4-vinyloxazolidin-2-one (7). A solution of $\mathrm{K}_{2} \mathrm{CO}_{3}(0.154 \mathrm{~g}, 1.10 \mathrm{mmol})$ in $\mathrm{MeOH}(10 \mathrm{~mL})$ was added to a solution of carbamate $\mathbf{6 i}$ $(0.389 \mathrm{~g}, 0.735 \mathrm{mmol})$ in $\mathrm{MeOH}(10 \mathrm{~mL})$ at $\mathrm{rt}$ under $\mathrm{N}_{2}$ atmosphere. The mixture was stirred for $2 \mathrm{~h}$. Water $(10 \mathrm{~mL})$ was added and the mixture was extracted with $\mathrm{CH}_{2} \mathrm{Cl}_{2}(3$ x $20 \mathrm{~mL}$ ). The combined organic phase was dried over $\mathrm{MgSO}_{4}$ and the solvent was evaporated under vacuum. The crude residue was purified by column chromatography (hexanes/EtOAc 3:2) to afford a mixture of debenzoylated products $(0.224 \mathrm{~g}, 86 \%$ yield). A solution of $\mathrm{TBAF} \cdot 3 \mathrm{H}_{2} \mathrm{O}(0.201 \mathrm{~g}, 0.62 \mathrm{mmol})$ and acetic acid $(35 \mu \mathrm{L}, 0.6$ mmol) in anhydrous THF (10 mL) was added via cannula to the mixture of debenzoylated products $(0.175 \mathrm{~g}, 0.41 \mathrm{mmol})$ in anhydrous THF $(10 \mathrm{~mL})$. The solution was stirred at $\mathrm{rt}$ for $5 \mathrm{~h}$. Phosphate buffer solution $(\mathrm{pH}=7,10 \mathrm{~mL})$ was added and the aqueous layer was extracted with EtOAc $(5 \times 20 \mathrm{~mL})$. The combined organic layer was dried over anhydrous $\mathrm{MgSO}_{4}$, and the solvent was evaporated under vacuum. The crude residue was purified by column chromatography $\left(\mathrm{CH}_{2} \mathrm{Cl}_{2} / \mathrm{MeOH}, 95: 5\right)$ to afford carbamate $7(0.060 \mathrm{~g}, 0.32 \mathrm{mmol})$ in $78 \%$ yield: colorless oil; $R_{f}($ EtOAc $)=0.35 ;[\alpha]_{\mathrm{D}}{ }^{25}$ $+31.5\left(c=1.0, \mathrm{CHCl}_{3}\right) ;{ }^{1} \mathrm{H} \mathrm{NMR}\left(400 \mathrm{MHz}, \mathrm{CDCl}_{3}\right) \delta 6.10(\mathrm{dd}, J=17.4,10.7 \mathrm{~Hz}, 1 \mathrm{H})$, $5.40(\mathrm{~d}, J=17.4,1 \mathrm{H}), 5.35(\mathrm{~d}, J=10.7 \mathrm{~Hz}, 1 \mathrm{H}), 4.49(\mathrm{~d}, J=8.8 \mathrm{~Hz}, 1 \mathrm{H}), 4.14(\mathrm{~d}, J=$ $8.8 \mathrm{~Hz}, 1 \mathrm{H}), 3.69-3.62(\mathrm{~m}, 4 \mathrm{H}), 3.37(\mathrm{~d}, J=8.5 \mathrm{~Hz}, 1 \mathrm{H}), 1.25(\mathrm{~d}, J=6.2 \mathrm{~Hz}, 3 \mathrm{H}) ;{ }^{13} \mathrm{C}$ NMR (101 MHz, $\left.\mathrm{CDCl}_{3}\right) \delta 135.2,116.1,78.4,74.1,69.2,64.5,20.9$; IR (film): 3379, 2922, 1737, 1396, 933, $721 \mathrm{~cm}^{-1}$; HRMS (ESI+) calculated for $\mathrm{C}_{8} \mathrm{H}_{14} \mathrm{NO}_{4}[\mathrm{M}+\mathrm{H}]^{+}=$ 188.0916 , found $=188.0917$. 
ACKNOWLEDGMENT. This work was supported by the Spanish Ministerio de Educación y Ciencia (CTQ2009-09692 and SAF2014-52223-C2-1-R), the University of Barcelona (fellowship to A.R.) and the Generalitat de Catalunya (2009SGR1037, 2014SGR107 and fellowship to C.S.)

\section{SUPPORTING INFORMATION}

${ }^{1} \mathrm{H}$ NMR spectra of the prepared starting materials allene $\mathbf{1}$ and chiral aldehydes. ${ }^{1} \mathrm{H}$ and ${ }^{13} \mathrm{C}$ NMR spectra of products $\mathbf{6 a - i}$, and 7. This material is available free of charge via the Internet at http://pubs.acs.org.

\section{AUTHOR INFORMATION}

Corresponding authors

*Fax: +34 933397878. Tel: +34 934021248. E-mail: xariza@ub.edu.

*Fax: +34 933397878. Tel: +34 934034819. E-mail: jordigarciagomez@ub.edu.

\section{REFERENCES AND FOOTNOTES}

(1) For a review on sphingofungins and related metabolites, see: Byun, H.-S.; Lu, X.; Bittman, R. Synthesis 2006, 2477.

(2) Shibasaki, M.; Kanai, M.; Fukuda, N. Chem. Asian J. 2007, 2, 20.

(3) Takahashi, A.; Kurasawa, S.; Ikeda, D.; Okami, Y.; Takeuchi, T. J. Antibiot. 1989, $42,1556$.

(4) For a review on the stereoselective construction of $\alpha, \alpha$-disubstituted $\alpha$-amino acids, see: (a) Cativiela, C.; Díaz-de-Villegas, M. D. Tetrahedron: Asymmetry 1998, 9, 3517. (b) Cativiela, C.; Díaz-de-Villegas, M. D. Tetrahedron: Asymmetry 2000, 11, 645; (c) Ohfune, Y.; Shinada, T. Eur. J. Org. Chem. 2005, 5127. (d) Kang, S. H.; Kang, S. Y.; 
Lee, H.-S.; Buglass, A. J. Chem. Rev. 2005, 105, 4537. (e) Cativiela, C.; Díaz-deVillegas, M. D. Tetrahedron: Asymmetry 2007, 18, 569.

(5) (a) Tendler, S. J. B.; Threadgill, M. D.; Tisdale, M. J. J. Chem. Soc., Perkin Trans. 1 1987, 2617. (b) Pedersen, M. L.; Berkowitz, D. B. J. Org. Chem. 1993, 58, 6966. (c) Berkowitz, D. B.; Jahng, W.-J.; Pedersen, M. L. Bioorg. Med. Chem. Lett. 1996, 6, 2151. (d) Berkowitz, D. B.; McFadden, J. M.; Chisowa, E.; Semerad, C. L. J. Am. Chem. Soc. 2000, 122, 11031.

(6) (a) Boumendjel, A.; Miller, S. P. F. Tetrahedron Lett. 1994, 35, 819. For a review on inhibitors of sphingolipid metabolism enzymes, see: (b) Delgado, A.; Casas, J.; Llebaria, A.; Abad, J. L.; Fabrias, G. Biochim. Biophys. Acta 2006, 1758, 1957. (c) Delgado, A.; Casas, J.; Llebaria, A.; Abad, J. L.; Fabrias, G. ChemMedChem 2007, 2, 580. (d) Sanllehí, P.; Abad, J. L.; Casas, J.; Delgado, A. Chem. Phys. Lipids 2016, 197, 69.

(7) (a) For the synthesis of protected 2-amino-2-vinyl-1,3-diols, see: Kumar, V.; Klimovica, K.; Rasina, D.; Jirgensons, A. J. Org. Chem. 2015, 80, 5934 and references therein. (b) For related 2-amino 2-vinyl alcohols, see: Cai, A.; Guo, W.; MartínezRodríguez, L.; Kleij, A. W. J. Am. Chem. Soc. 2016, 138, 14194 and references therein. (8) Boyer, J.; Allenbach, Y.; Ariza, X.; Garcia, J.; Georges, Y. Vicente, M. Synlett 2006, 1895.

(9) Ariza, X.; Cornellà, J.; Font-Bardia, M.; Garcia, J.; Ortiz, J.; Sánchez, C.; Solans, X. Angew. Chem., Int. Ed. 2009, 48, 4202.

(10) Isomerization of $\mathbf{3}$ to $\mathbf{5}$ is an equilibrium process in which the cyclic carbamate $\mathbf{5}$ is clearly favored in basic media and other non-hydrolytic solvents tested. Related basecatalyzed rearrangement processes involving $\mathrm{N}$-acyl or $\mathrm{N}$-alkyl cyclic carbamates have previously been reported in the literature: (a) Roush, W. R.; Adam, M. A. J. Org. Chem. 
1985, 50, 3752. (b) Boger, D. L.; Ledeboer, M. W.; Kume, M. J. Am. Chem. Soc. 1999, 121, 1098. (c) Bew, S. P.; Bull, S. D.; Davies, S. G.; Savory, E. D.; Watkin, D. J. Tetrahedron 2002, 58, 9387.

(11) Horino, Y.; Kimura, M.; Tanaka, S.; Okajima, T.; Tamaru, Y. Chem. Eur. J. 2003, 9, 2419.

(12) For a similar migration, see: McCombie, S. W.; Nagabhushan, T. L. Tetrahedron Lett. 1987, 28, 5395.

(13) The relative stereochemistry was correlated with the known stereochemistry of tosylated products 3 (ref. 9). Thus, $O$-benzoylated products 6 were $N$-tosylated to afford the doubly protected carbamates and the corresponding $N$-tosylated products 3 were $O$ benzoylated to yield the same compound.

(14) Michel, P.; Ley, S. V. Angew. Chem. Int. Ed. 2002, 41, 3898.

(15) Massad, S. K.; Hawkins, L. D.; Baker D. C. J. Org. Chem. 1983, 48, 5180. 